\title{
Comparative toxicity of gold preparations in treatment of rheumatoid arthritis
}

\author{
J. S. LAWRENCE \\ From Medizinische Hochschule Hannover, Abt. für Balneologie und Rheumatologie im Niedersäch Staatsbad \\ Nenndorf, W. Germany
}

\begin{abstract}
Lawrence, J. S. (1976). Annals of the Rheumatic Diseases, 35, 171-173. Comparative toxicity of gold preparations in treatment of rheumatoid arthritis. Patients with rheumatoid arthritis have been treated alternately with aurothioglucose and aurothiomalate. In the earlier part of the study an oily suspension of aurothioglucose and an aqueous solution of aurothiomalate were used, but later an aqueous solution of aurothioglucose was alternated with the oily suspension and an oily suspension of aurothiomalate with the aqueous solution. Skin eruptions, stomatitis, and albuminuria were significantly more common in patients treated with the aqueous solution than with the oily suspension.
\end{abstract}

Although a number of gold compounds have been used in the treatment of rheumatoid arthritis, few comparisons have been made of their relative toxicity. Oily suspensions were introduced around 1933 in an attempt to produce a more even concentration of gold in the tissues but clinicians tended to avoid such preparations for fear that excretion might be delayed in the event of side effects arising. It is now known that excretion is very slow whatever preparation is used, and it must be considered whether slow absorption, by avoiding peaks in the blood levels, may not be more advantageous. Rothermich, Bergen, and Philips (1967) have shown that where gold is given at 2- or 3-week intervals in maintenance therapy the plasma gold level becomes negligible if a soluble salt is used but the decline is avoided if an oil-depot preparation is used.

\section{Method}

Patients with rheumatoid arthritis attending a rehabilitation clinic were allocated consecutively to one of four treatment groups.

(1) Sodium aurothiomalate in aqueous solution.

(2) Sodium aurothionalate in oily suspension.

(3) Aurothioglucose in oily suspension.

(4) Aurothioglucose in aqueous solution.

Dosage in the four groups was identical, varying from 50 to $200 \mathrm{mg}$ weekly, being based on the activity of the disease as determined by the plasma fibrinogen level (Lawrence, 1953). When the plasma fibrinogen, estimated monthly, fell below $450 \mathrm{mg} / 100 \mathrm{ml}$ on three consecutive occasions a maintenance dose of $50 \mathrm{mg}$ every second week was given. Results were assessed after two years' treatment.

\section{Results}

The disease was considered to have become quiescent when joint pain and swelling had subsided and the plasma fibrinogen level had returned to normal. No significant difference was observed in the 2-year assessment between the four treatment groups with regard to the number becoming quiescent or improved (Table). The erythrocyte sedimentation rate attained normal levels and remained so for at least 3 months in $60 \%$ of those on an aqueous preparation and in $62 \%$ on an oily suspension. There was thus no difference in therapeutic effectiveness between oily and aqueous preparations.

Toxicity, on the other hand, showed very significant differences. Skin eruptions were four times as common in those treated with aqueous preparations, stomatitis three times, and albuminuria more than five times as common in this group. The greater toxicity with the aqueous preparations applied both to those treated with aurothiomalate and with aurothioglucose, and the skin eruptions were significant with the former.

\section{Discussion}

The number of patients treated with aqueous aurothioglucose and oily aurothiomalate is small 
since discontinuance of aqueous aurothioglucose preparation by the manufacturers put a stop to the trial. The results, however, already showed a greater toxicity from this preparation and are confirmed significantly by the comparison between aqueous and oily aurothiomalate. In addition to the side effects shown in the Table, two patients developed purpura, one on oily aurothioglucose, the other on aqueous aurothiomalate. The condition subsided in 2 weeks. One patient on aqueous aurothiomalate developed peripheral neuritis. Apart from these 3, and one patient on aqueous aurothiomalate in whom dermatitis and stomatitis persisted for more than 4 months after stopping treatment, it was possible to resume the gold injections at a lower dose level.

Most skin reactions to gold and probably also stomatitis and albuminuria must be regarded as immune reactions in which gold acts as a hapten, since these reactions may occur at any stage of the treatment and are unrelated to levels of excretion (Lawrence, 1961; Gottlieb, Smith, and Smith, 1972). In the present series side effects occurred after a total dosage varying from 0.03 to $4 \mathrm{~g}$ and the range was comparable in the various treatment groups.

\section{References}

Gottlieb, N. L., Smith, P. M., AND SMIth, E. M. (1972) Arthr. and Rheum., 15, 582 (Gold excretion correlated with clinical course during chrysotherapy of R.A.)

LAWRENCE, J. S. (1953) Ann. rheum. Dis., 12, 129 (Factors in gold dosage and toxicity) (1961) Ibid., 20, 341 (Studies with radioactive gold)

Rothermich, N. O., Bergen, W., AND PhinIPS, V. K. (1967) Arthr. and Rheum., 10, 308 (Use of plasma gold levels in determining dose frequency, type of gold salt and impending toxicity) 